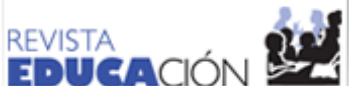

Revista Educación

ISSN: 0379-7082

ISSN: 2215-2644

revedu@gmail.com

Universidad de Costa Rica

Costa Rica

\section{Deserción estudiantil en la educación superior: reflexiones sobre la gestión enfocada en la retención o la permanencia}

Pereira Santana, Adrián; Vidal Cortez, Marcela

Deserción estudiantil en la educación superior: reflexiones sobre la gestión enfocada en la retención o la permanencia

Revista Educación, vol. 45, núm. 1, 2021

Universidad de Costa Rica, Costa Rica

Disponible en: http://www.redalyc.org/articulo.oa? $\mathrm{id}=44064134011$

DOI: https://doi.org/10.15517/revedu.v45i1.40602

Esta obra está bajo una Licencia Creative Commons Atribución-NoComercial-SinDerivar 3.0 Internacional. 


\title{
Deserción estudiantil en la educación superior: reflexiones sobre la gestión enfocada en la retención o la permanencia
}

\author{
Higher Education Dropouts. Reflections about Managing Retention or Persistence
}

Adrián Pereira Santana

Universidad Miguel de Cervantes, Chile

apereira@corp.umc.cl

(iD http://orcid.org/0000-0002-5849-6446

Marcela Vidal Cortez

Universidad Miguel de Cervantes, Chile

marcela.vidal@alumni.umc.cl

(DD http://orcid.org/0000-0002-9579-8515
DOI: https://doi.org/10.15517/revedu.v45i1.40602

Redalyc: http://www.redalyc.org/articulo.oa?id=44064134011
Recepción: 13 Marzo 2020

Aprobación: 05 Agosto 2020

\section{Resumen:}

La preocupación por la deserción académica en la educación superior es un tema de particular importancia en la gestión de instituciones de educación superior [IES], estableciéndose una tensión entre deserción y retención, entendiéndola como resultado de las acciones que desarrolla la institución para que el estudiantado se mantenga y progrese académicamente hasta su graduación. Ello implica la comprensión de la calidad educativa vinculada a la gestión de retención que efectúan las instituciones, y es dicho concepto lo que los sistemas evalúan. Sin embargo, a partir de la década de 2000, cobran mayor relevancia las visiones que promueven acciones orientadas a la permanencia, reconociendo el rol central del estudiantado en su proceso formativo y en la decisión de continuar estudios, en que la generación de vínculos con la institución y con el sistema en general aparecen capitales; ello junto al cuestionamiento del concepto de deserción, al evidenciar estudios el reingreso de un porcentaje importante de desertores, al sistema en el corto y mediano plazo. El estudio, que persigue problematizar la tensión entre deserción-retención mediante la revisión de la literatura acerca los factores de deserción, con foco en el periodo posterior al 2000 y sus implicancias para las concepciones tradicionales convoca a desarrollar una propuesta integradora de estas perspectivas que entran en tensión de manera diferenciada con el proceso de deserción, una desde el accionar organizacional, otra desde el espacio vincular afectivo del alumnado y su compromiso y motivación con el proceso, cuando el efecto de la deserción o permanencia, responde a una gestión acerca de lo organizacional, el apoyo en lo personal, y la definición final de cada estudiante, ya sea de forma temporal o permanente.

Palabras Clave: Deserción Académica, Educación Superior, Permanencia, Retención.

\section{Abstract:}

Concern about students dropping out of higher education institutions is a particularly stressful administrative issue for higher education institutions. Educational systems must assess efforts made by institutions to keep students enrolled until they progress academically and graduate understanding that educational quality is linked to managing retention. However, since 2000, there has been a greater focus on promoting actions for students to stay in school, by acknowledging that they play a key role in their own educational development and deciding to continue their studies, thus, highlighting the importance of strengthening links with the university and the educational system, as a whole. The concept of students dropping out of school is also reconsidered since studies have shown that many students do, in fact, return to school in the short and medium-terms. This study analyzes the administrative stress associated with students dropping out of school vs retention based on a literature review, specifically after the year 2000 that looks at the factors that impact a student's decision to drop out of school and how this impacts traditional views permanently. As a result, a comprehensive and differentiated approach is required to address the dropout issue which involves organizational action, the need for students to establish and emotional connection with their studies, attain personal support and the student's final decision by to drop out either temporarily or permanently.

KEYWORDS: School dropout, Higher Education, Permanence, Retention. 


\section{INTRODUCCIÓN}

La pregunta por la deserción estudiantil y los resultados expresados habitualmente como retención han generado una preocupación en cada una de las Instituciones de Educación Superior (IES). Ello implicó, en un comienzo, el reconocimiento de las IES de su rol en la gestión de las herramientas institucionales y el apoyo en el fortalecimiento de los elementos individuales del estudiantado, y por lo tanto la activación de políticas, planes y programas que incrementen la retención.

Así, se establece una díada entre deserción y retención en la gestión de las IES en relación con esta temática. Se entiende que los índices de deserción académica y los expresados como retención institucional, se han vuelto un referente central en la evaluación de las IES en general y de las universidades en lo particular, estableciendo una centralidad de dichos indicadores en el sistema.

A modo de ejemplo, el Ministerio de Educación de Chile [MINEDUC] a través de su sistema de información de educación superior (SIES) respecto de las Universidades calcula y presenta datos para el sistema y para cada una de las IES de Retención al primer y segundo año de estudios, en programas de 4 y 5 años (Subsecretaria de Educación, 2019).

Sin embargo, esta preocupación tiende a invisibilizar el componente humano del estudiantado, enfocándose en la no deserción o retiro, como resultado de un proceso externo, institucional; mientras un modelo que se base en estudiantes, poniendo al centro la persona del o la estudiante, lo visualiza desde la perspectiva de este último, analizando la permanencia como factor que fomenta la progresión y la graduación.

Ello no implica que las gestiones que desarrolla la institución no sean consideradas o ponderadas en términos de la labor que implica para la permanencia del estudiantado al interior de las instituciones de educación superior, sino más bien, centra de mejor forma la toma de decisión respecto de la gestión general de la institución en la entrega de los servicios educacionales, en términos de calidad y pertinencia de las acciones que promueven la permanencia de estudiantes de manera directa, sin ser únicamente restringidos a la labor de la institución para evitar que el alumnado se retire, sino y sobre todo las acciones que permiten que este se vincule y mantenga en la institución o el sistema.

Si bien en Chile se han establecido como estándares de evaluación, la deserción/retención de primer y segundo año, estudios recientes (Acción Educar, 2019; Ministerio de Educación Chile [Mineduc] (2019) problematizan acerca de la pertinencia de dicha medición, en tanto, el reingreso al sistema del estudiantado que ha desertado se acerca al 50\% tras tres años, lo cual hace variar de forma importante dicho indicador.

En este sentido, el alumnado que deserta provisoriamente, en el conjunto de características (individuales, institucionales y relacionales) que diversas investigaciones, que son revisadas en este artículo, han establecido como elementos que conforman la etiología de la deserción, no queda claramente representado en la perspectiva de análisis de la tensión deserción-retención, y requiere de una nueva categorización que dé cuenta de la efectiva vinculación con el proceso formativo que enfrenta en el proceso de desarrollo de su carrera profesional incorporando en la gestión que se efectúa en las IES, por ejemplo, los cambios vocacionales o de institución académica.

Es por ello, que el presente análisis tiene por objetivo problematizar la tensión entre deserción-retención, a la vez que analizar la pertinencia de la instalación de una díada retención-permanencia, que refleje no solo de los resultados de las acciones de las IES para retener al estudiantado, sino las que se ejecutan para que se vincule de manera efectiva y afectiva, aunque presente lagunas en su trayectoria formativa. Ello en la medida que los autores se desenvuelven en una IES de tipo inclusiva, en que el alumnado presenta múltiples factores de riesgo, que implicaría una baja probabilidad de desarrollo de trayectorias educativas exitosas y que, sin embargo, presentan resultados en términos de retención y graduación, titulaciones similares a otras instituciones del sistema, incluso de tipo selectivas.

Para ello, se establece una descripción general de la metodología de revisión de fuentes secundarias, seguida de la revisión de los principales antecedentes teóricos a partir de 2000 y con foco en la década 2010-2019, 
respecto de la discusión acerca de deserción y retención estudiantil, las principales causas establecidas y su relación con los mecanismos de gestión, para a partir de aquellos, configurar las bases que permitan establecer la discusión en torno a la pertinencia de modificar la tensión existente en el análisis desde la deserciónretención, como díada contrapuesta, a la tensión retención-permanencia como díada de gestión, elemento que configura las conclusiones de este análisis.

\section{Metodología}

El presente artículo considera una revisión de fuentes secundarias a partir del año 2000, con un foco en la producción posterior a 2010 y hasta la actualidad en la temática de deserción universitaria, para ello se buscó en bases de datos de corriente principal, con foco en indexaciones SciELO (Scientific Electronic Library Online), Scopus y WoS (Web of Science).

La selección de los artículos tuvo como criterio la saturación de la información respecto a los factores de riesgo de deserción presentes en la literatura especializada, como manera de abordar comprensivamente el estado del arte acerca de la discusión en torno a la deserción, retención, permanencia en las IES.

La selección del periodo corresponde a la determinación de la discusión actual, y se incluyeron fuentes anteriores en caso de que estas corresponden a elementos que sustentan la discusión posterior, o bien que son referentes para la problematización del fenómeno.

La discusión se desenvuelve fundamentalmente en el ámbito teórico, y las cifras estadísticas presentadas, corresponden a insumos para la discusión o sustentación de las afirmaciones que modifican la interpretación tradicional de los conceptos tratados en el presente artículo.

\section{ANTECEDENTES TEÓRICOS}

La revisión de la literatura especializada da cuenta de una preocupación transversal en el sistema educacional, vinculado a los procesos de ingreso, desarrollo de trayectorias académicas, rendimiento y eficiencia del sistema, en todo el ámbito de la educación superior (Acción Educar, 2019; Eckert y Suénaga, 2015).

Esta preocupación ha implicado que las Universidades generen propuestas de abordaje que permitan responder a la deserción, desde la retención, tras el desarrollo de procesos de análisis y reflexión, que respondan a los contextos y necesidades de sus estudiantes (Hanne y Mainardi, 2013), así se han establecido múltiples metodologías las cuales buscan mantener al estudiantado en el sistema.

Tal relevancia ha logrado este asunto, que la retención estudiantil constituye hoy uno de los indicadores fundamentales de eficiencia de las organizaciones educacionales, transformándose en un indicador de calidad (Pineda, Pedraza y Moreno, 2011). Ello en el entendido que el efecto de la deserción da cuenta también de una merma social, que ocurre al abandonar la formación inicial, lo que implica la generación de efectos tanto económicos como personales, tales como frustración y descontento (Matheu, Ruff, Ruiz, Benites y Morong, 2018).

Así, la deserción es entendida como el abandono del sistema, sea este permanente o transitorio, ya sea por características personales, o de desempeño académico, (Zavala, Álvarez, Vázquez, González y Bazán, 2018). No obstante, esta definición varía de acuerdo con quien la ejecuta, y a partir de la reincorporación al sistema, se vuelve también compleja (Pérez, 2019).

Este último factor, la reincorporación, se vuelve relevante, ya que la deserción de un programa puede implicar un giro vocacional (cambio de carrera o institución), o bien un afrontamiento de una dificultad particular, así, un estudio del MINEDUC (2019) da cuenta que "del 28,8 \% de estudiantes de pregrado de la cohorte 2015 que desertaron en el primer año, un 49,1 \% reingresó a la educación superior en el corto plazo (dentro de los tres años siguientes a su deserción)” (p. 4). En este sentido, la deserción se vuelve un 
tema complejo, tanto por su resultado, como también por la diversidad de perspectivas, de estrategias y de mecanismos de gestión, que dan lugar a una amplia tipología de tipos de deserción (Henríquez y Escobar, 2016). Así, en Chile, un informe de 2013, indica que tres de cada diez estudiantes abandonan su institución durante el primer curso, con alguna variabilidad entre tipo de IES (Navarrete, Candia y Puchi, 2013).

Barrero (2015) hace un listado de autores que dan cuenta de la problemática, desde fines de los $90 \mathrm{y}$ comienzos de la década del 2000, en términos de las repercusiones sociales —Páramo y Correa (2012)—, fracaso personal y consecuencias sociales - Gómez (1998) —, así como en la dimensión institucional y de políticas públicas - Ramírez (2002) — . Así, Barrero (2015) da cuenta de la necesidad de interpretar del fenómeno en una perspectiva sistémica que incorpora los aspectos tanto micro como macrosociales, y en todo su trayecto.

Todo lo indicado, evidencia la relevancia de este asunto para las Universidades, lo que implica el reconocimiento de las diversas formas de deserción - permanente o transitoria (Canales y De los Ríos, 2007) - de forma de establecer mejores planes de apoyo que respondan a los diversos factores de riesgo que presenta el estudiantado de una determinada institución (Barragán y Patiño, 2013).

Uno de los elementos que ha sido consignado como una de las causales de preocupación en las Universidades, respecto de la retención-deserción, está relacionada con las políticas que han fomentado la ampliación de la cobertura universitaria (Hanne y Mainardi, 2013; Canales y De los Ríos, 2009; Henríquez y Escobar, 2016; Palmeros, 2014).

En Latinoamérica, Hanne y Mainardi (2013), analizaron la configuración de las Universidades e indicaron que desde sus orígenes fueron concebidas como instituciones elitistas, monolíticas y homogeneizantes, las cuales producto de la demanda de mayor pertinencia social y cultural y cobertura de matrícula, generaron un cambio en el sistema, complejizándolo, cuestión que es concordante con la realidad en Chile, la cual ha experimentado un crecimiento y modificación de su estructura, brindando acceso a segmentos de población que antes no lo hacían (Canales y De los Ríos, 2009) a través de la incorporación de las IES privadas a partir de 1981, aumentando el número de estudiantes (Henríquez y Escobar, 2016).

A este respecto, Castillo y Gamboa (2012) plantean que los sistemas educativos en el continente han producido esfuerzos para asegurar calidad al incorporar el carácter inclusivo que implica aceptar la diversidad de estudiantes que reciben, lo cual implicaría una pérdida de autonomía y un desgaste en el ejercicio docente para los cuerpos académicos en las IES entre otros efectos para los agentes e individuos involucrados (Patiño y Cardona 2012).

Este aumento de cobertura observado en Latinoamérica ha permitido que estos nuevos segmentos de población accedan, sin embargo, en condiciones de mayor vulnerabilidad, tanto económica como académica, al incluir en ella a estudiantes provenientes de los primeros quintiles de ingreso (Canales y De los Ríos, 2009; Palmeros, 2014). A este respecto, el Instituto Nacional de Estadísticas de Chile en 2003 señalaba que un porcentaje relevante de estudiantes era primera generación en cursar estudios superiores (Canales y De los Ríos, 2009).

Así, la preocupación por la calidad en la educación superior, tras la incorporación de nuevos perfiles de estudiantes después de la aplicación de las políticas de aumento de cobertura y aquellas que proponen la educación superior como derecho - como las orientadas a la gratuidad — han implicado el desafío de garantizar el acceso para todo el estudiantado, como también su implicación en los procesos (Palmeros, 2014), que permitan el desarrollo de aprendizajes (Palmeros y García, 2017) que de acuerdo con los lineamientos curriculares actuales, sean significativos (Ausubel, 1963) y situados (Lave, y Wenger, 1991).

Ello ha implicado, para el sistema y para cada IES, la consideración de la inclusión educativa como un desafío permanente, ya sea mediante programas de acceso especiales, o bien mediante definiciones institucionales, todo lo que requiere la conceptualización, reconocimiento y planeación del ingreso, considerando las características del alumnado y sus necesidades (Palmeros, 2014), pues el nuevo estudiantado 
requiere del aprendizaje de nuevos códigos prácticos y simbólicos en relación con quienes cuentan con capital cultural que considera dichos componentes en su formación previa (Canales y De los Ríos, 2009).

Ello en el entendido de que estudiantes que no cuentan con el capital cultural, al formar parte de grupos sociales que son frecuentemente excluidos y en condiciones de no participación (autoexclusión), presentan mayor probabilidad de deserción estudiantil, lo que da cuenta que se está frente a un fenómeno social complejo, de características que implican la reflexión en torno a la estigmatización, la exclusión y la necesaria consideración de estudios en profundidad de los entramados culturales asociados a este fenómeno (Barrero, 2015).

También se ha analizado la teoría costo beneficio para explicar la deserción, considerando que la educación tendría un efecto movilizador social con beneficios a largo plazo, el costo de oportunidad de desarrollar estudios en comparación a otras actividades, y el costo asociado al bienestar y salud emocional, que es un elemento de tensión, sobre todo en estudiantes vulnerables y frente a enfermedades propias o de familiares (Díaz, 2008). Así, el costo percibido, no responde de forma lineal a un solo objeto.

En términos cuantitativos, el SIES de MINEDUC plantea que, en Chile en 2016, la deserción alcanzó un 22,50\%, cifras que empeoran al considerar los programas e-learning, b-learning y MOOC donde la deserción alcanza hasta el 90\% (Rodríguez, Espinoza, Ramírez y Ganga, 2018).

En la actualidad esta realidad no ha variado de manera relevante, así lo manifiesta el Ministerio de Educación de Chile (MINEDUC, 2019), cuando señala que la deserción de estudiantes en primer año oscila entre el $26 \%$ y el $31 \%$ en el periodo comprendido entre 2008 y 2017. Estas cifras son elevadas en análisis internacionales, y desfavorecen fundamentalmente al estudiantado de los quintiles de ingresos más bajos (Canales y De los Ríos, 2009).

\section{DisCuSión TEÓRICA DE LOS FACTORES}

Entendiendo la complejidad de la materia, los modelos de análisis que implican la determinación de factores asociados o predictores de riesgo de deserción, varían en tanto énfasis y enfoques analíticos, así, desde la psicología se enfatizan aspectos individuales y de relación con la institución, mientras que, desde la sociología, se desarrolla en términos de variables de contexto (familia, entorno social, etc.) y su relación con las variables individuales y de sistema (Himmel, 2002).

Así, se han revisado una serie de modelos y teorías, que, bajo perspectivas explicativas o predictivas, pueden agrupar los factores personales, ya sea en su preparación académica, aspectos psicológicos, características familiares y socioeconómicas (Matheu, et al., 2018).

Por su parte, los enfoques económicos, analizan la deserción desde una perspectiva de racionalidad costo beneficio, en que el alumnado analiza la inversión de recursos (tiempo, dinero, esfuerzo, etc.) en determinados habitus educacionales (Bordieu y Passeron, 1979), y los consiguientes beneficios de corto mediano y largo plazo, en términos de expectativas, en relación con actividades alternativas en el periodo de tiempo de los estudios (Himmel, 2002).

Paralelamente, los enfoques denominados organizacionales focalizan la labor de retención en las instituciones de educación superior, entendiéndola como la capacidad de influir en aquellos elementos que explican la deserción, así como de compensar los costos asociados a la educación terciaria, de manera que el estudiantado se mantenga y concluya sus estudios. Algunos referentes de esta línea son Tinto, Bean, Chapman, Pascarella o Weidman (Himmel, 2002).

Henríquez y Escobar (2016), respecto de este último enfoque, indican que aquí surge la concepción de retención académica, y como los factores asociados a beneficios estudiantiles, la existencia de actividades extracurriculares, de existencia de recursos y número de estudiantes por docente impactan de forma relevante en su retención. En esta misma línea, se ubican las investigaciones que ha elaborado el Ministerio de Educación de Chile, y que correlacionan la retención con la acreditación institucional. 
Si bien es cierto que la temática se ha transversalizado en el sistema, debido a su magnitud (Acción Educar, 2019), en relación con los factores que explican la deserción en instituciones de educación superior, aún no existe un consenso que dé cuenta de los factores que componen la etiología de la deserción-retención, sin embargo, hay algunos ejes articuladores los permiten posibles agrupaciones categoriales.

En este sentido encontramos propuestas que plantean que las causales se dividen entre personales y del sistema (Acción Educar, 2019), mientras otras las dividen entre aquellas relacionadas a factores de integración social y causas relacionadas a rendimiento académico (Canales y De los Ríos, 2007) y que focalizan, como otros autores (Eckert y Suénaga, 2015; Soria y Zúñiga, 2014; Navarrete et al., 2013) en el estudiantado de primer año.

En esta línea el Ministerio de Educación de Chile, señala que los factores asociados a la deserción de primer año consideran "factores vocacionales, económicos, financieros, familiares, de rendimiento académico o de salud, entre otros, y que pueden llevar a un estudiante a abandonar sus estudios" (MINEDUC, 2019, p. 7).

$\mathrm{Al}$ respecto, en la definición de las principales características que devienen en factores en la etiología de la deserción académica, el tránsito en el ingreso a la universidad aparece como relevante (Eckert y Suénaga, 2015; Soria y Zúñiga, 2014), así, los resultados en enseñanza media (Navarrete et al., 2013), los resultados en pruebas estandarizadas y los resultados académicos de asignaturas en primer año cobran relevancia, concordante con estudios que establecen una relación entre los resultados de primer año, con los generales en los procesos académicos (Eckert y Suénaga, 2015).

Este tránsito en primer año puede dar cuenta también de una perspectiva de mayor alcance temporal, así lo hace por ejemplo Suárez y Díaz (2015), al vincular la deserción estudiantil en el nivel terciario al estrés académico de primer año.

Sin embargo, la estructuración de factores individuales o del sistema educacional no parecen ser suficientes al incorporar la variable socioeconómica o contextual, en que la relación no es únicamente estudiante-sistema de educación superior, sino en una mixtura de relaciones, así factores de índole microsistémico como los de orden familiar, tales como soporte afectivo, cambios en la composición de la familia (nacimiento y/o muertes), enfermedades, etc.; mesosistémicos, como pérdida del empleo (propio o del jefe de hogar), soportes sociales, etc.; devienen en varios casos en el abandono - temporal o definitivo - del sistema educativo (Canales y De los Ríos, 2009).

Así lo refieren una serie de investigaciones las cuales han dado cuenta de otros factores que correlacionan el abandono o deserción con factores individuales tales como falta de vocación, problemas de salud, económicos; factores mixtos entre lo institucional y las expectativas de cada estudiante, tal como no conformidad con el programa, o bien el rendimiento académico; y factores de índole social como influencia de pares o familia (Barrero, 2015, Centro Interuniversitario de Desarrollo [CINDA], 2017) y en términos culturales, en tanto saberes previos y disposiciones culturales que les permitan desempeñarse en los espacios académicos (Hanne y Mainardi, 2013), concordante con lo referido previamente en términos del dominio de códigos prácticos y simbólicos (Canales y De los Ríos, 2009). Así se vuelve necesario reconocer que estudiantes con itinerarios formativos dificultados, como pueden ser estudiantes pertenecientes a comunidades originarias (con marcos culturales propios) o con algún tipo de discapacidad, cuentan con dificultades adicionales para el desarrollo de una carrera universitaria (Hanne y Mainardi, 2013).

En cuanto al componente familiar, se abordan dos aspectos, el primero de ellos referido al apoyo, acerca del cual se concluye que, a mayor soporte y apoyo, es mayor la probabilidad de éxito en términos de egreso y titulación (Suárez y Díaz, 2015), mientras que una familia que no da soporte ni apoyo, aumenta la sensación de constituir la educación en un elemento sacrificable dentro del proyecto de vida. En segundo término, y parte de multiplicidad de estudios en la materia, el nivel de educación de los padres (Barragán y Patiño, 2013; Suárez y Díaz, 2015; Leyton, Vásquez y Fuenzalida, 2012), el que correlaciona sobre todo en el caso de la educación de la madre, respecto de la cual la probabilidad de deserción disminuye a mayor nivel educativo de los padres. 
En relación con el establecimiento de proveniencia, para el caso chileno, se establece que el mayor porcentaje de retención y prematrícula corresponde a estudiantes provenientes de establecimiento particulares pagados (4,4\% de deserción permanente), mientras que, en el extremo opuesto, la deserción en estudiantes provenientes de educación municipal (16,5\% de deserción permanente) es casi cuatro veces más que en el primer caso (MINEDUC, 2019)

Por otra parte, factores de tipo individual como el compromiso con los estudios (Fernández, Martínez y Melipillán, 2009), de orden interaccional, como el compromiso institucional y calidad institucional (Bean, 1980, citado en Canales y De los Ríos, 2007) permiten complementar las interpretaciones que buscan integrar un modelo de análisis que dé cuenta de la complejidad observada, incorporando en la etiología de la deserción, una serie de factores de deserción-permanencia, en dimensiones académicas, institucionales, personales y sociodemográficas (Barrero, 2015).

Por su parte, la OCDE establece que las tasas de éxito educativo se correlacionan con puntajes en pruebas de admisión y tipo de establecimiento educacional de origen, ambos muy relacionados con el nivel socioeconómico del estudiantado, y que dan cuenta de la inequidad del sistema educativo (Canales y De los Ríos, 2009)

Respecto de los factores individuales, ellos corresponden a características tales como el género, la edad, discapacidad (Palmeros, 2014), establecimiento educacional de origen, la condición de trabajador, además de las de orden psicológico, tales como las motivacionales, emocionales, adaptativas (Zavala et al., 2018).

Así, por ejemplo, con respecto al género, un estudio que analiza la cohorte 2015 (MINEDUC, 2019), da cuenta que las mujeres muestran menores porcentajes de deserción que los varones, con diferencias de hasta 5 puntos porcentuales. Asimismo, presentan mayor cantidad de reingreso (casi 4 puntos porcentuales), lo que implica una cantidad de desertores permanentes superior en los varones, lo cual se podría relacionar con la alta tolerancia a la frustración que presentan estudiantes mujeres en relación con los hombres (Rodríguez et al., 2018).

En términos de edad, a mayor rango etario, aumenta la deserción permanente, así lo demuestra el estudio desarrollado por el Ministerio de Educación de Chile (2019), el indica que en estudiantes del rango 15-19 años la deserción permanente es de 6,6 \% en contraposición al $25 \%$ de los que superan los 24 años. Esta situación es concordante con la condición de trabajador, quienes observan una menor integración social y académica, así como menor contacto y vínculo social con sus compañeras y compañeros, lo que los hace más vulnerables a la deserción (Leyton, et al., 2012).

En términos académicos, problemas cognitivos, rendimiento, métodos de estudio o dificultades universitarias en términos de adaptación por tipo de enseñanza (Zavala et al., 2018) también constituyen un factor relevante en la deserción, el cual requiere un tratamiento específico de orden institucional.

Respecto de la situación de discapacidad, la vulnerabilidad viene dada por características propias de su situación y las diferentes necesidades que presentan estas y estos estudiantes (Palmeros, 2014).

En términos psicológicos, los factores más recurrentes en la literatura corresponden a factores vocacionales y de falta de motivación, la insatisfacción de expectativas (Zavala et al., 2018), la confianza en sí mismo (Donoso, Donoso y Arias, 2010; Suárez y Díaz 2015), autoestima y autoconcepto (Fernández, Martínez y Melipillán, 2009) estos dos últimos identificados como factores de persistencia.

En dicho sentido, es necesario entender la motivación académica como una conducta orientada al logro estudiantil (Montes, Almonacid, Gómez, Zuluaga y Tamayo, 2014), en esta dimensión, se pueden englobar los elementos ligados a la autoestima y autoconcepto los que vistos desde una perspectiva positiva devienen factores de permanencia, mientras que en la perspectiva del desgano, afecta tanto el rendimiento como la adaptabilidad a la vida universitaria, transformándose en factores de riesgo de deserción (Carranza y Apaza, 2015).

Todos estos factores devienen en lo que Martínez (2009) interpreta como factores de desesperanza o indefensión aprendida, situación en la cual el afrontamiento de situaciones complejas posiciona al 
estudiantado en un contexto de incapacidad de reacción, abandonando la tarea al no tener un elemento motivacional a la base que le permita avanzar y concluir sus estudios, al asumir que independiente de las acciones que ejecutan, el resultado será el fracaso.

En cuanto a los factores organizacionales, aparecen aquellos que dicen relación con las acciones que efectúa la institución en pro de la continuidad de estudios (Zavala et al., 2018), entendiendo por ello tanto las formas de docencia (Peña, 2019; Zavala et al., 2018), los horarios (Suárez y Díaz, 2015) y mecanismos que promueven su retención, tanto en términos de apoyos académicos, como también económicos (Zavala et al., 2018; Arancibia y Trigueros, 2018).

Así, elementos tales como la infraestructura deficiente, recursos humanos percibidos como deficientes, falta de planificación de las y los docentes, tratos inadecuados (Zavala et al., 2018) e incompatibilidad horaria para desarrollar los estudios (Suárez y Díaz, 2015) aparecen como preponderantes.

Estos factores se transforman en espacios de mayor riesgo de deserción en tanto la propuesta institucional es limitada, en aspectos tales como el desarrollo académico y el financiamiento (Zavala et al., 2018), ello en atención a las expectativas que aparecen en estudiantes en los procesos de admisión fundamentalmente.

Así, respecto a los beneficios económicos, Acción Educar (2019) en un estudio para Chile, indica que las posibilidades de deserción varían en torno al $15 \%$ entre estudiantes sin beneficios estudiantiles, en relación con quienes cuentan con ellos.

Otro de los elementos, corresponde a la incompatibilidad horaria con otras acciones, por ejemplo, del tipo laboral, extensiones de jornada o cambios en el empleo, implican la imposibilidad de continuidad de estudios (Suárez y Díaz, 2015). Si bien este factor puede ser externo (laboral), instituciones que presentan flexibilidad al respecto permiten mayores niveles de retención.

En dicho sentido, la estructuración de la oferta académica también impacta en la deserción estudiantil. Es conocido que las áreas de conocimiento y tipo de carrera presentan diferencias importantes en las tasas de deserción, por ejemplo, el estudio del SIES (MINEDUC, 2019) establece diferencias importantes entre las áreas de Administración y Comercio, con valores que rondan el 10\% de deserción permanente, mientras que en las ciencias básicas el valor alcanza un 2,3\%.

En último término aparecen los factores de orden relacional o bilateral, en los cuales el énfasis se da en dos dimensiones fundamentales, la que dice relación con los estilos de enseñanza (Zavala et al., 2018; Peña, 2019) y los de integración (Canales y De los Ríos, 2007; Leyton et al., 2012; Palmeros, 2014).

En el primer orden, los estilos y metodologías de docencia que establecen la interacción entre docentes y sus estudiantes (Zavala et al., 2018) y que se dispone en el aula, deviene en factor protector frente a la deserción, mientras que las deficiencias pedagógicas y la no consideración de las necesidades de estos, aumenta el riesgo (Peña, 2019).

Ello se complejiza con estudiantes con condiciones específicas de vulnerabilidad como la discapacidad, en que la falta de formación al respecto, de las tipologías de la discapacidad y sus requerimientos, así como la ausencia o deficiencia de recursos para el aprendizaje (Palmeros, 2014).

En cuanto a la integración, ello dice relación con la posibilidad de ser y sentirse parte del sistema o de la institución, al respecto, Canales y De los Ríos (2007) plantean que la deserción responde a un fenómeno de desintegración en el sentido durkheimiano.

Este sentimiento de desintegración se puede ver acrecentado, cuando existen aulas diversas, en que estudiantes vulnerables se sienten en desventaja frente a sus compañeras y compañeros (González y Uribe, 2002), lo cual implica la imposibilidad de establecer lazos (Suárez y Díaz 2015) al visibilizar su proyecto a través de su origen, comparándolo con los demás (Leyton et al., 2012), estableciendo una brecha que se aprecia imposible de salvar. En estudiantes con características de vulnerabilidad evidente, tales como los discapacitados, esta situación deviene en segregación, visualización y exacerbación de la diferencia con el consiguiente rechazo (Palmeros, 2014). 
Esta situación no es solo frente a los compañeros, sino con el sistema en general, que requiere reconocer sus redes en términos de recursos y apoyos (Suárez y Díaz, 2015) que implican un desafío institucional en términos de políticas que tomen en cuenta las diferencias en necesidades del estudiantado (Palmeros, 2014)

Esta desintegración social, da cuenta a la vez de dos aspectos que dicen relación con las acciones de acogida en los ámbitos académicos y sociales al interior de las instituciones de educación superior, las expectativas generadas al comienzo de los estudios, y el ambiente universitario (Zavala et al., 2018). Respecto de este último, las interacciones que promueve una institución en los procesos académicos, pero también fuera de ellas, permiten la implicación de estudiantes con sus pares, generando ambientes de confianza que fomentan la colaboración y convivencia, promoviendo la mantención en el sistema (Zavala et al., 2018).

Tal como se ha señalado, la consideración de la inclusión educativa como un desafío para las IES, considerando las necesidades, características y capital cultural del alumnado (Palmeros, 2014; Canales y De los Ríos, 2009). Ha implicado la generación de acciones de apoyo en la inserción académica, tanto para el desarrollo general, como también para suplir bajos niveles de preparación académica (Henríquez y Escobar, 2016; Zavala et al., 2018), trasladando la preocupación desde el acceso a la retención, favoreciendo la conclusión efectiva de los estudios de quienes acceden a la educación superior (Pérez, 2019).

Ello basado en estudios que dan cuenta que estudiantes que reciben apoyo académico durante el primer año, tienen mayor probabilidad de continuar los estudios (Rodríguez et al., 2018), cuestión más relevante en estudiantes que son primera generación, o sin padres universitarios y que por tanto no disponen del capital cultural equivalente a quienes cuentan con familias con formación en educación superior (Canales y De los Ríos, 2007).

Ya en los años 70 y 80 se comienza a manifestar la necesidad de generar ajustes al sistema de educación superior, en términos de caracterizar el perfil de ingreso de las y los estudiantes, con el objeto de conocerlos y retenerlos, lo que implicaba la generación de programas orientados al efecto (Díaz, 2008).

Si bien el foco aparece analizado desde la perspectiva académica, la deserción y permanencia ha sido también enfocada desde las lógicas del vínculo, situación que apunta a la generación de los programas que se han instalado, sobre todo en instituciones privadas (Palmeros, 2014); y que se basan en que la permanencia se relaciona con "los vínculos que se establecen a través de la vida” (Valdés, 2018 p. 333).

En general, estos programas, son comprendidos como aquellos que buscan garantizar el acompañamiento del estudiantado durante su trayectoria educativa, entregando herramientas necesarias para el término de los estudios y el desarrollo de competencias y habilidades del egreso (Pineda et al., 2011), e incorporan elementos vinculados a la nivelación de conocimientos, inserción universitaria, técnicas de estudio y/o desarrollo personal que permitan la preparación académica y colaborar en suplir las falencias socioafectivas que afectan la retención (Henríquez y Escobar, 2016).

También, se ha incorporado en esta perspectiva las condiciones que las instituciones disponen para sus estudiantes, considerando la serie de recursos con que cuenta de forma directa en la gestión de la deserción, como de tipo institucional. Así, se destacan los programas, recursos, modelos y prácticas, sistemas de apoyo, y cómo estos factores se imbrican entre sí (Matheu, et al., 2018) y en particular la vinculación entre docentes y estudiantes, así como sus expectativas, interés o entusiasmo (Peña, 2019; Matheu et al., 2018).

En dicho sentido, la Universidad de Chile, en el año 2018, establece seis categorías de acciones para retener al alumnado: programas de apoyo académico, programas de apoyo económico y social, programa de interés y motivación, programas de orientación y asesoría psicológica, programas de apoyo a docentes y programas de análisis comparado de estrategias de retención (Barrero, 2015).

Así, estudios que se han desarrollado entre los años de 1970 y 2007, dan cuenta que aquellos programas que incorporan estrategias de nivelación, técnicas de estudio y desarrollo personal, se correlacionan de manera importante con la no deserción, con especial impacto en estudiantes que cuentan con alta vulnerabilidad, la que va asociada de manera frecuente con baja preparación académica y falencias socioafectivas (Henríquez y Escobar, 2016). 
Todos estos antecedentes permiten establecer un continuo de desarrollo en la construcción de alternativas de abordaje de la deserción en instituciones de educación superior. Este continuo está conformado por la perspectiva retención $\mathrm{v} /$ la perspectiva de la permanencia.

La deserción, ha sido vista desde una doble consideración, que se ha identificado desde la lógica de retención y persistencia, centrándose la primera en las acciones o esfuerzos institucionales para mantener al estudiantado en la IES hasta su graduación, mientras que la segunda se enfoca en aquellos elementos de voluntad y motivación que hacen a un/a estudiante mantenerse en la institución durante su trayectoria académica (Valdés, 2018).

Así, respecto a las estrategias que se orientan a disminuir la deserción, encontramos en un primer momento aquellas que pretenden hacerse cargo de los factores institucionales, es decir, los elementos que parecen ser propios de la gestión de la institución, y los elementos de tipo individual que pudiesen estar descendidos en el estudiantado.

De este modo, el enfoque de Tinto, reseñado en Matheu et al. (2018), pareciera entender desde un comienzo en un continuo entre retención y persistencia, lo que implica que el rol de las IES está dado en el primero de estos enfoques.

Estos enfoques - de retención - tienen en su base el componente economicista de la transacción, lo cual pone el problema de la deserción en torno a la inestabilidad de los ingresos institucionales (Díaz, 2008), lo cual implica la necesidad de las instituciones de aumentar la retención (Henríquez y Escobar, 2016).

Por ello es necesario trasladar la preocupación de la responsabilidad institucional de la retención, lo que implica mover el foco de preocupación desde la comprensión de las motivaciones de deserción, hacia las acciones que se pueden ejecutar para que el estudiantado se vincule y mantenga en la institución (Matheu, et al. 2018).

Un cambio de perspectiva que instale la discusión en la permanencia implica la creación de vínculos entre institución y estudiantes, generando relaciones mutuas de confianza, en que el rol docente es fundamental, conociendo las necesidades e intereses de sus estudiantes, planteando los procesos en función de ellos (Vázquez y Escámez, 2010).

En este sentido, el de la mantención como resultado de la interacción entre estudiante e institución, es que la reivindicación de la teoría del vínculo de Pichón Rivière (citado en Valdés, 2018) cobra nueva relevancia, poniendo el foco en la relación, la cual se encuentra siempre en movimiento y en un tránsito dialéctico adentro-afuera, en lo que denomina interacción dialéctica, y que da cuenta de un constante equilibrio en la permanencia de estudiantes y los contextos, en un análisis de factores de riesgo y protección (vinculación) en torno a la problemática de la deserción.

\section{Conclusiones}

Tras la revisión de la teoría y en la construcción del entramado de sentidos que conforma la trayectoria educativa en educación superior, propio del proceso de problematización perseguido en el presente trabajo, se conforma una serie de relaciones que conforman la etiología de la deserción y la determinación de sus factores está altamente desarrollada, quedando la labor a las instituciones de caracterizar de acuerdo con sus perfiles de estudiantes, aquella conformación particular que permitiría mejorar la gestión de casos en cada una de las realidades.

Sin embargo, la determinación de la problemática en las instituciones de educación superior está dada por la necesaria tensión establecida en términos del estudiantado que abandona el sistema de manera permanente (desertores) y en el debate aparecen dos conceptos que responden a la gestión que cada una de las instituciones realiza para abordar el fenómeno de la deserción. Estos conceptos son el de retención y el de permanencia. 
Si bien es cierto, que ambos conceptos se han utilizado de manera alternada, como si se tratara de sinónimos en el accionar institucional acerca de la deserción, ambos cuentan con una carga semántica diferenciada, tanto en el ejecutor de la acción (IES) como en estudiantes en condición de posible deserción.

Por tanto, la distinción semántica aparece como un aspecto que dista de ser baladí, en tanto la gestión que desarrollan las IES tienen un propósito, que es reducir la cantidad de estudiantes que desertan del sistema por situaciones que se alejen de lo vocacional, razón por la cual despliegan una serie de acciones tendientes a apoyar, disminuir, o incluso resolver los problemas que se transforman en factores de riesgo de deserción entre sus estudiantes.

De esta forma, la oferta de una serie de medidas tendientes a la retención, corresponde a una perspectiva que se puede denominar de primera generación, que parte con las reflexiones acerca de la necesidad de mantener al estudiantado dentro del sistema y que se corresponde a la perspectiva principal con la que la temática se instala en el sistema, incluso como medidor de la calidad de una institución, pero que aparece como una responsabilidad desde la institución y que invisibiliza la vinculación que permite la mantención en el sistema.

Una segunda generación del pensamiento acerca de la retención corresponde a las perspectivas que emergen en la década del 2000 y que, desde los aspectos motivacionales, la desesperanza, la vinculación y el continuo entre integración y desintegración, comienzan a cuestionar de forma más bien tácita, los modelos de deserción-retención y plantear uno de deserción-permanencia.

Esta mirada complejiza la gestión de la deserción en las IES, pero mantiene ambas perspectivas de forma aislada, hay acciones de retención y acciones vinculadas a la permanencia, no observándose una perspectiva que las integre.

Así, la necesaria comprensión de que la deserción es un espacio en que se juegan diversos factores en la conformación de una etiología de la deserción, implica reconocer la articulación de sentidos y voluntades en torno a la retención-permanencia que ejecuta por una parte la institución y por la otra el estudiantado. Ello implica reconocer también, que la determinación última será siempre del alumnado, por lo que el foco, más que en la retención, debiese estar puesto en la permanencia.

Este cambio, reconoce la centralidad del grupo estudiantil en su proceso de formación profesional, su autonomía, a la vez que sitúa las acciones de la institución al servicio de este tomador de decisión, en una mirada que articula el continuo entre los procesos de enseñanza y los procesos de aprendizaje, en los cuales median las acciones que la institución desarrolla y promueve en pro de la integración, disminución de los factores de riesgo y fortalecimiento del vínculo en estudiantes, promoviendo en ellos la voluntad de permanecer en el sistema.

Esta situación es además congruente con los últimos estudios, que permiten reconocer la diferencia entre la deserción temporal y permanente, y visualizar - al menos en el caso de Chile — que un porcentaje importante de quienes desertan en el sistema (hasta el $50 \%$ ) retornan en el corto y mediano plazo (hasta tres años posterior al retiro). Esta suspensión prolongada, no permanente, aumenta los registros de deserción artificiosamente, sin considerar aspectos tales como cambios vocacionales, cambios de institución, o afrontamiento de problemas personales/familiares, que dan cuenta de una perseverancia tan importante como aquellos que elaboran sus estudios de manera continua.

En este plano, el de la continuidad/discontinuidad, también se vuelve importante el proceso de integración/vinculación, no solo con la institución que acoge al estudiantado en su primer paso por la educación superior, sino también en cuanto sistema. En el primer caso pensando en que retorne a la carrera o institución, en el segundo caso, en cuanto considere la posibilidad de retomar estudios en otras instituciones o en otras vocaciones, con la proyección de concluir su trayectoria formativa, aunque sea postergada.

En este sentido, aparece necesario ajustar la nomenclatura de aquellas acciones que realizan las IES en la gestión de la deserción, orientándolas al proceso de permanencia más que al de retención. De esta forma, la centralidad del estudiantado que declara la mayoría de los modelos curriculares actuales encuentra un correlato en la relación que se establece entre la organización y sus estudiantes. 
Ello no quiere decir que se abandone el foco de la retención, sino más bien que se integre en un análisis más complejo, en que las lógicas de retención que subyacen una perspectiva económica y de prestación servicios, se encuentre dentro de las alternativas posibles, pero supeditadas a la gestión de la vinculación efectiva y afectiva de las y los estudiantes con su proceso formativo en educación superior.

Ello implica que la gestión al interior de las IES debe considerar una oferta flexible de acuerdo con las necesidades de sus estudiantes, y que se responda a los problemas de capital cultural, formativos y socio afectivos de quienes desarrollan programas académicos en la organización, promoviendo la generación de planes de apoyo durante toda la trayectoria formativa, aunque con un foco en los primeros años, y planes especiales de reincorporación o reingreso, que permitan una vinculación bidireccional con el proceso formativo, en que IES y estudiante conformen una asociación en pro del logro de la progresión y consecuente graduación/titulación.

Todo ello implica a la vez un cambio comprensivo en términos de calidad, ya no evaluada en términos de deserción - retención, sino en términos de permanencia - progresión, que incorpore las estadísticas de reingreso al sistema, IES, carrera y/o programa, como antecedentes que dan cuenta de una vinculación positiva en torno a la formación de profesionales, y no establezca un indicador que a la luz de la evidencia, enfoca este aspecto de la calidad en un resultado que puede ser transitorio, y por ende no dar cuenta de la realidad de la problemática.

Con ello, la concepción de calidad educativa se perfecciona al incorporar una estadística más precisa, al establecer la diversidad de trayectorias formativas que se producen en las IES y que permiten la mejor comprensión de la educación superior en su diversidad de proyectos educativos y en la diversidad de estudiantes que participan del sistema.

Esta situación implica la necesidad de seguir analizando la situación, mediante análisis longitudinales, de mayor alcance, que permitan dar cuenta efectiva de las trayectorias que reingresan al sistema de manera recurrente, o en plazos superiores a los analizados en los sistemas de gestión; esto permitirá que las IES tengan herramientas más pertinentes para este tipo de estudiantes.

\section{Referencias Bibliográficas}

Acción Educar (2019). Financiamiento de la Educación Superior y sus efectos en la Retención Estudiantil. Recuperado de https://bit.ly/2VGpJ4Z

Arancibia, R. y Trigueros, C. (2018). Aproximaciones a la deserción universitaria en Chile. Educação e Pesquisa, 44, e165743. doi: https://doi.org/10.1590/s1678-4634201708165743

Ausubel, D. (1963). The psychology of meaningful verbal learning. New York: Grune and Stratton.

Barrero, F. (2015). Investigación en deserción estudiantil universitaria: educación, cultura y significados. Revista Educación y Desarrollo Social, 9(2), 86-101. doi: https://doi.org/10.18359/reds.948

Barragán, D. y Patiño, L. (2013) Elementos para la comprensión del fenómeno de la deserción universitaria en Colombia. Más allá de las mediciones. Cuadernos Latinoamericanos de Administración, 9(16), 55-66. doi: http s://doi.org/10.18270/cuaderlam.v9i16.1248

Bordieu, P. y Passeron, J. (1979). La Reproducción. Barcelona: Editorial Laia, S.A.

Canales, A. y De los Ríos, D. (2007). Factores Explicativos de la Deserción Universitaria. Calidad en la Educación, (26), 173-201. doi https://doi.org/10.31619/caledu.n26.239

Canales, A. y De los Ríos, D. (2009). Retención de estudiantes vulnerables en la educación universitaria. Revista Calidad en la Educación, 30, 50-83. doi https://doi.org/10.31619/caledu.n30.173

Carranza, R. y Apaza, E. (2015). Autoconcepto académico y motivación académica en jóvenes talento de una universidad privada de Tarapoto. Propósitos y Representaciones, 3(1), 233-263: doi, https://doi.org/10.20511/ pyr2015.v3n1.72 
Castillo, M. y Gamboa, R. (2012). Desafíos de la educación en la sociedad actual. Revista Electrónica Diálogos Educativos, 12(24), 55-69. Recuperado de http://revistas.umce.cl/index.php/dialogoseducativos/article/view/ 1055

Centro Interuniversitario de Desarrollo [CINDA] (2017) Evaluación de Logro de Perfiles de Egreso: Experiencias Universitarias. Santiago: Copygraph

Díaz, C. (2008). Modelo conceptual para la deserción estudiantil universitaria chilena. Estudiospedagógicos (Valdivia), 34(2), 65-86: doi, https://doi.org/10.4067/S0718-07052008000200004

Donoso, S., Donoso, G. y Arias, Ó. (2010). Iniciativas de retención de estudiantes de educación superior. Calidad En La Educación, (33), 15-61. doi https://doi.org/10.31619/caledu.n33.138

Eckert, K. y Suénaga, R. (2015). Análisis de deserción-permanencia de estudiantes universitarios utilizando técnica de clasificación en minería de datos. Formación Universitaria, 8(5), 3-12. doi: https://doi.org/10.4067/S0718-50 062015000500002

Fernández, O., Martínez, M. y Melipillán, R. (2009). Estrategias de aprendizaje y autoestima: su relación con la permanencia y deserción universitaria. Estudios Pedagógicos(Valdivia), 35(1), 27-45: doi, https://doi.org/10.40 67/S0718-07052009000100002

González, L. y Uribe, D. (2002). Estimaciones sobre la "repitencia” y deserción en la educación superior chilena. Consideraciones sobre sus implicaciones. Calidad En La Educación, (17), 75-90. doi: https://doi.org/10.3161 9/caledu.n17.408

Hanne, A. y Mainardi, A. (2013). Reflexiones sobre la inclusión de grupos en situación de vulnerabilidad en la educación superior. El Dispositivo Tutorial: un espacio en construcción. Revista de Docencia Universitaria, 11(2), 173-192. doi: https://doi.org/10.4995/redu.2013.5572

Henríquez, N. y Escobar, D. (2016). Construcción de un modelo de alerta temprana para la detección de estudiantes en riesgo de deserción de la Universidad Metropolitana de Ciencias de la Educación. Revista mexicana de investigación educativa, 21(71), 1221-1248. Recuperado de https://bit.ly/2VJHKzs

Himmel, E. (2002). Modelos de análisis de la deserción estudiantil en la educación superior. Calidad de la Educación, (17), 91-108: doi, https://doi.org/10.31619/caledu.n17.409

Lave, J. y Wenger, E. (1991). Situated learning: Legitimateperipheral participation. New York: Cambridge University Press.

Leyton, D., Vásquez, A. y Fuenzalida, V. (2012). La experiencia de estudiantes de contextos vulnerables en diferentes Instituciones de Educación Superior Universitaria (IESU): Resultados de investigación. Calidad en la educación, (37), 61-97. doi: https://doi.org/10.4067/S0718-45652012000200003

Martínez, H. (2009). Autopercepción social y atribuciones cognoscitivas en estudiantes de bajo rendimiento académico. Electronic Journal of Research in Education Psychology, 7(19). doi https://doi.org/10.25115/ejrep.v $7 \mathrm{i} 19.1327$

Matheu, A., Ruff, C., Ruiz, M., Benites, L. y Morong, G. (2018). Modelo de predicción de la deserción estudiantil de primer año en la Universidad Bernardo O'Higgins. Educação e Pesquisa, 44, 1-23. doi: https://doi.org/10.15 90/S1678-4634201844172094

Ministerio de Educación de Chile [MINEDUC]. (2019). Deserción de primer año y Reingreso a la Educación Superior en Chile. Análisis de la Cohorte 2015. Chile: Gobierno de Chile, SIES. Recuperado de https://bit.ly/2PJz8VK

Montes, I., Almonacid, P., Gomez, S., Zuluaga, F. y Tamayo, E. (2014). Análisis de la Deserción Estudiantil en los Programa de Pregrado de la Universidad EAFIT. Cuadernos de Investigación EAFIT, (10-10), 1-117. doi: http s://doi.org/10.2139/ssrn.2427487

Navarrete, S., Candia, R. y Puchi, R. (2013). Factores asociados a la deserción/retención de los estudiantes mapuche de la Universidad de la Frontera e incidencia de los programas de apoyo académico. Calidad En La Educación, (39), 43-80, doi: https://doi.org/10.4067/s0718-45652013000200003

Palmeros, G. (Coord.), (2014). Capítulo IV: Discapacitados. En Gairín, J., Castro, D. y Rodríguez, D. (Ed.). Acceso, permanencia y egreso en la universidad de colectivos vulnerables en Latinoamérica: intervenir y cambiar la realidad. Barcelona: Santillana del Pacífico. Recuperado de https://ddd.uab.cat/record/123666 
Palmeros, G. y García, A. (2017). Inclusión Educativa y Grupos Vulnerables. Un Análisis de los Estudiantes de la DAEA. Perspectivas Educativas, 28(64), 58-64. doi: https://doi.org/10.19136/pd.a28n64.2129

Páramo, G. y Correa, C. (2012). Deserción estudiantil universitaria. Conceptualización. Revista Universidad EAFIT, 35(114), 65-78. Recuperado de https://bit.ly/3bPs7el

Patiño, L.; y Cardona, A. (2012). Revisión de algunos estudios sobre la deserción estudiantil universitaria en Colombia y Latinoamérica. Theoria 21(1). Recuperado de http://revistas.ubiobio.cl/index.php/RT/article/view/1241/1 192

Peña, A. (2019). Los factores pedagógicos influyen en la deserción universitaria. INNOVA Research Journal, 4(3), 108115, doi: https://doi.org/10.33890/innova.v4.n3.2019.996

Pérez, T. (2019) Deserción en la Cohorte 2018 en la Escuela de Ciencia Política y Administración Pública. Santiago, Chile: Universidad Miguel de Cervantes.

Pineda, C., Pedraza, A. y Moreno, I. (2011). Efectividad de las estrategias de retención universitaria: la función del docente. Educación y Educadores, 14(1). Recuperado de https://bit.ly/2ziiibm

Rodríguez, A., Espinoza, J., Ramírez, L. y Ganga, A. (2018). Deserción Universitaria: Nuevo Análisis Metodológico. Deserción Universitaria, 11(6), 107-118. doi: https://doi.org/10.4067/S0718-50062018000600107

Soria, K. y Zúñiga, S. (2014). Aspectos determinantes del éxito académico de estudiantes universitarios. Formación Universitaria, 7(5), 41-50. doi https://doi.org/10.4067/S0718-50062014000500006

Suárez, N. y Díaz, L. (2015) Estrés académico, deserción y estrategias de retención de estudiantes en la educación superior. Revista de Salud Pública, 17(2), 300-313. doi: https://doi.org/10.15446/rsap.v17n2.52891

Subsecretaria de Educación (2019). Mifuturo.cl: Buscador de instituciones, Chile. Recuperado de https://www.mifut uro.cl/buscador-de-instituciones/

Valdés, C. (2018). "Deserción” universitaria: entre desvinculaciones institucionales y búsqueda de sentidos de vida. Revista Latinoamericana de Ciencias Sociales, Niñez y Juventud, 16(1), 31-344. doi: https://doi.org/10.11600 /1692715x.16120

Vázquez, V. y Escámez, J. (2010). La profesión docente y la ética del cuidado. Revista Electrónica de Investigación Educativa, 12(SPE), 1-17. Recuperado de http://www.scielo.org.mx/pdf/redie/v12nspe/v12nspeal.pdf

Zavala, M., Álvarez, M., Vázquez, M., González, I. y Bazán, A. (2018). Factores Internos, Externos y Bilaterales asociados con la deserción en estudiantes universitarios. Interacciones, 4(1), 59-69. doi: https://doi.org/10.240 16/2018.v4n1.103

\section{BY-NC-ND}

